\title{
Characterizations of Transversal and Fundamental Transversal Matroids
}

\author{
Joseph E. Bonin \\ Department of Mathematics \\ The George Washington University \\ Washington, D.C. 20052, USA \\ jbonin@gwu . edu
}

\author{
Joseph P. S. Kung \\ Department of Mathematics \\ University of North Texas \\ Denton, TX 76203, USA \\ kung@unt .edu
}

\author{
Anna de Mier* \\ Departament de Matemàtica Aplicada II \\ Universitat Politècnica de Catalunya \\ Jordi Girona 1-3, 08034 \\ Barcelona, Spain \\ anna.de.mier@upc.edu \\ Submitted: Sep 17, 2010; Accepted: Apr 29, 2011; Published: May 8, 2011 \\ Mathematics Subject Classification: 05B35
}

\begin{abstract}
A result of Mason, as refined by Ingleton, characterizes transversal matroids as the matroids that satisfy a set of inequalities that relate the ranks of intersections and unions of nonempty sets of cyclic flats. We prove counterparts, for fundamental transversal matroids, of this and other characterizations of transversal matroids. In particular, we show that fundamental transversal matroids are precisely the matroids that yield equality in Mason's inequalities and we deduce a characterization of fundamental transversal matroids due to Brylawski from this simpler characterization.
\end{abstract}

\section{Introduction}

Transversal matroids can be thought of in several ways. By definition, a matroid is transversal if its independent sets are the partial transversals of some set system. A result of Brylawski gives a geometric perspective: a matroid is transversal if and only if it has an affine representation on a simplex in which each union of circuits spans a face of the simplex.

*Partially supported by Projects MTM2008-03020 and Gen. Cat. DGR 2009SGR1040. 
Unions of circuits in a matroid are called cyclic sets. Thus, a set $X$ in a matroid $M$ is cyclic if and only if the restriction $M \mid X$ has no coloops. Let $\mathcal{Z}(M)$ be the set of all cyclic flats of $M$. Under inclusion, $\mathcal{Z}(M)$ is a lattice: for $X, Y \in \mathcal{Z}(M)$, their join in $\mathcal{Z}(M)$ is their join, $\operatorname{cl}(X \cup Y)$, in the lattice of flats; their meet in $\mathcal{Z}(M)$ is the union of the circuits in $X \cap Y$. The following characterization of transversal matroids was first formulated by Mason [13] using sets of cyclic sets; the observation that his result easily implies its streamlined counterpart for sets of cyclic flats was made by Ingleton [9]. Theorem 1.1 has proven useful in several recent papers $[1,2,3]$. For a family $\mathcal{F}$ of sets we shorten $\cap_{X \in \mathcal{F}} X$ to $\cap \mathcal{F}$ and $\cup_{X \in \mathcal{F}} X$ to $\cup \mathcal{F}$.

Theorem 1.1 A matroid is transversal if and only if for all nonempty sets $\mathcal{F}$ of cyclic flats,

$$
r(\cap \mathcal{F}) \leq \sum_{\mathcal{F}^{\prime} \subseteq \mathcal{F}}(-1)^{\left|\mathcal{F}^{\prime}\right|+1} r\left(\cup \mathcal{F}^{\prime}\right) .
$$

It is natural to ask: which matroids satisfy the corresponding set of equalities? We show that $M$ satisfies these equalities if and only if it is a fundamental transversal matroid, that is, $M$ is transversal and it has an affine representation on a simplex (as above) in which each vertex of the simplex has at least one matroid element placed at it. The main part of this paper, Section 4, provides four characterizations of these matroids.

We recall the relevant preliminary material in Section 2. Theorems 4.1 and 4.4 give new characterizations of fundamental transversal matroids; from the former, two other new characterizations (Theorem 4.5 and Corollary 4.6) follow easily. The proofs of Theorems 4.1 and 4.4 use a number of ideas from a unified approach to Theorem 1.1 and a second characterization of transversal matroids (the dual of another result of Mason, from [14]); we present this material in Section 3 and deduce another of Mason's results from it. We conclude the paper with a section of observations and applications; in particular, we show that Brylawski's characterization of fundamental transversal matroids [5, Proposition 4.2] follows easily from the dual of Theorem 4.1.

As is common, we assume that matroids have finite ground sets. However, no proofs use finiteness until we apply duality in Theorem 5.2, so, as we spell out in Section 5, most of our results apply to matroids of finite rank on infinite sets.

We assume basic knowledge of matroid theory; see [15, 16]. Our notation follows [15]. A good reference for transversal matroids is [4]. It is easy to see that proving the results in this paper in the case of matroids that have no loops immediately yields the same results for matroids in general. Since, in addition, the geometric perspective on transversal matroids that conveys most insight into key parts of our work fits best with matroids that have no loops, we focus on loopless matroids in this paper.

We use $[r]$ to denote the set $\{1,2, \ldots, r\}$.

\section{Background}

Recall that a set system $\mathcal{A}$ on a set $S$ is a multiset of subsets of $S$. It is convenient to write $\mathcal{A}$ as $\left(A_{1}, A_{2}, \ldots, A_{r}\right)$ with the understanding that $\left(A_{\sigma(1)}, A_{\sigma(2)}, \ldots, A_{\sigma(r)}\right)$, where $\sigma$ is any 
permutation of $[r]$, is the same set system. A partial transversal of $\mathcal{A}$ is a subset $I$ of $S$ for which there is an injection $\phi: I \rightarrow[r]$ with $x \in A_{\phi(x)}$ for all $x \in I$. Transversals of $\mathcal{A}$ are partial transversals of size $r$. Edmonds and Fulkerson [8] showed that the partial transversals of a set system $\mathcal{A}$ on $S$ are the independent sets of a matroid on $S$; we say that $\mathcal{A}$ is a presentation of this transversal matroid $M[\mathcal{A}]$.

Of the following well-known results, all of which enter into our work, Corollary 2.3 plays the most prominent role. The proofs of some of these results can be found in [4]; the proofs of the others are easy exercises.

Lemma 2.1 Any transversal matroid $M$ has a presentation with $r(M)$ sets. If $M$ has no coloops, then each presentation of $M$ has exactly $r(M)$ nonempty sets.

Lemma 2.2 If $M$ is a transversal matroid, then so is $M \mid X$ for each $X \subseteq E(M)$. If $\left(A_{1}, \ldots, A_{r}\right)$ is a presentation $M$, then $\left(A_{1} \cap X, \ldots, A_{r} \cap X\right)$ is a presentation of $M \mid X$.

Corollary 2.3 If $\left(A_{1}, A_{2}, \ldots, A_{r}\right)$ is a presentation of $M$, then for each $F \in \mathcal{Z}(M)$, there are exactly $r(F)$ integers $i$ with $F \cap A_{i} \neq \emptyset$.

Lemma 2.4 For each $A_{i} \in \mathcal{A}$, its complement $A_{i}^{c}=E(M)-A_{i}$ is a flat of $M[\mathcal{A}]$.

Lemma 2.5 If $\left(A_{1}, A_{2}, \ldots, A_{r}\right)$ is a presentation of $M$ and if $x$ is a coloop of $M \backslash A_{i}$, then $\left(A_{1}, A_{2}, \ldots, A_{i-1}, A_{i} \cup x, A_{i+1}, \ldots, A_{r}\right)$ is also a presentation of $M$.

Corollary 2.6 For any presentation $\left(A_{1}, A_{2}, \ldots, A_{r}\right)$ of a transversal matroid $M$, there is a presentation $\left(A_{1}^{\prime}, A_{2}^{\prime}, \ldots, A_{r}^{\prime}\right)$ of $M$ with $A_{i} \subseteq A_{i}^{\prime}$ and $A_{i}^{\prime c} \in \mathcal{Z}(M)$ for $i \in[r]$.

A presentation $\left(A_{1}, A_{2}, \ldots, A_{r}\right)$ of $M$ is maximal if, whenever $\left(A_{1}^{\prime}, A_{2}^{\prime}, \ldots, A_{r}^{\prime}\right)$ is a presentation of $M$ with $A_{i} \subseteq A_{i}^{\prime}$ for all $i \in[r]$, then $A_{i}=A_{i}^{\prime}$ for all $i \in[r]$. It is well known that each transversal matroid of rank $r$ has a unique maximal presentation with $r$ sets.

A transversal matroid is fundamental if it has a presentation $\left(A_{1}, A_{2}, \ldots, A_{r}\right)$ for which no difference $A_{i}-\bigcup_{j \in[r]-i} A_{j}$, for $i \in[r]$, is empty. Clearly any transversal matroid can be extended to a fundamental transversal matroid: whenever a set in a given presentation is contained in the union of the others, adjoin a new element to that set and to the ground set, but to no other set in the presentation.

In the next paragraph we describe how, given a presentation of a transversal matroid $M$, we get an affine representation of $M$ on a simplex. What we describe, which is based on [5], is a special case of affine representations of matroids in general (see [15, Sections 1.5 and 6.2]); however, these particular affine representations of transversal matroids can be seen as very direct geometric encodings of presentations. To keep the focus on this aspect, we describe only where the elements of $M$ are placed on the simplex relative to the vertices and to each other (any assignment of coordinates that meets these conditions will give an affine representation of $M$ in the sense of [15, Section 1.5]).

Recall that a simplex $\Delta$ in $\mathbb{R}^{r-1}$ is the convex hull of $r$ vectors, $v_{1}, v_{2}, \ldots, v_{r}$, that are affinely independent. The faces of $\Delta$ are the convex hulls of the subsets of $\left\{v_{1}, v_{2}, \ldots, v_{r}\right\}$ and so can be 


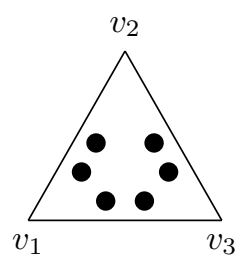

(a)

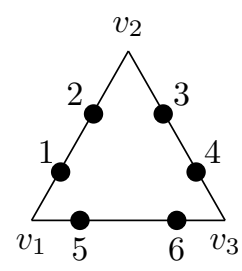

(b)



(c)

Figure 1: Three representations of the uniform matroid $U_{3,6}$ on the simplex with vertices $v_{1}, v_{2}, v_{3}$.

identified with these subsets. Given a presentation $\mathcal{A}=\left(A_{1}, A_{2}, \ldots, A_{r}\right)$ of $M$ and a simplex $\Delta$ with vertices $v_{1}, v_{2}, \ldots, v_{r}$, for $x \in E(M)$, let $\Delta_{\mathcal{A}}(x)$ be the face $\left\{v_{k}: x \in A_{k}\right\}$; also, for $X \subseteq E(M)$, let $\Delta_{\mathcal{A}}(X)$ be the face $\cup_{x \in X} \Delta_{\mathcal{A}}(x)$. (We will omit the subscript $\mathcal{A}$ when only one presentation is under discussion.) Note that if $F \in \mathcal{Z}(M)$, then $|\Delta(F)|=r(F)$ by Corollary 2.3, so $F=\{x: \Delta(x) \subseteq \Delta(F)\}$. Given $\mathcal{A}$, to get the corresponding affine representation of $M$, first extend $M$ to a fundamental transversal matroid $M^{\prime}$ by extending $\mathcal{A}$ to a presentation $\mathcal{A}^{\prime}$ of $M^{\prime}$, as above. We get an affine representation of $M^{\prime}$ by, for each $x \in E\left(M^{\prime}\right)$, placing $x$ as freely as possible (relative to all other elements of $M^{\prime}$ and to the vertices) in the face $\Delta_{\mathcal{A}^{\prime}}(x)$ of $\Delta$. Thus, a cyclic flat $F$ of $M^{\prime}$ of rank $i$ is the set of elements in some face of $\Delta$ with $i$ vertices. The affine representation of $M$ is obtained by restricting that of $M^{\prime}$ to $E(M)$. Note that, by construction, such an affine representation of $M$ can be extended to an affine representation of a fundamental transversal matroid by adding elements at the vertices of $\Delta$.

Such representations of the uniform matroid $U_{3,6}$ for the presentations (a) ([6], [6], [6]), (b) $(\{1,2,5,6\},\{1,2,3,4\},\{3,4,5,6\})$, and (c) $(\{1,4,5,6\},\{2,4,5,6\},\{3,4,5,6\})$ are shown in Figure 1. (Only elements $x$ with $\Delta(x) \neq\left\{v_{1}, v_{2}, v_{3}\right\}$ are labelled in the figure.)

Note that the presentation can be recovered from the placement of the elements. The following result of Brylawski [5] extends these ideas.

Theorem 2.7 A matroid $M$ is transversal if and only if it has an affine representation on a simplex $\Delta$ in which, for each $F \in \mathcal{Z}(M)$, the flat $F$ is the set of elements in some face of $\Delta$ with $r(F)$ vertices.

With this result, we can give a second perspective on fundamental transversal matroids. A basis $B$ of a matroid $M$ is a fundamental basis if each $F \in \mathcal{Z}(M)$ is spanned by $B \cap F$. In any affine representation of a matroid $M$ with a fundamental basis $B$, if the elements of $B$ are placed at the vertices of a simplex $\Delta$, then a cyclic flat of rank $i$ is the set of elements in some $i$-vertex face of $\Delta$. It follows from Theorem 2.7 that a matroid is a fundamental transversal matroid if and only if it has a fundamental basis.

We use the following terminology from ordered sets, applied to the lattice $\mathcal{Z}(M)$ of cyclic flats. An antichain in $\mathcal{Z}(M)$ is a set $\mathcal{F} \subseteq \mathcal{Z}(M)$ such that no two sets in $\mathcal{F}$ are related by inclusion. A filter in $\mathcal{Z}(M)$ is a set $\mathcal{F} \subseteq \mathcal{Z}(M)$ such that if $A \in \mathcal{F}$ and $B \in \mathcal{Z}(M)$, and if $A \subseteq B$, then $B \in \mathcal{F}$. An ideal in $\mathcal{Z}(M)$ is a set $\mathcal{F} \subseteq \mathcal{Z}(M)$ such that if $B \in \mathcal{F}$ and $A \in \mathcal{Z}(M)$, and if $A \subseteq B$, then $A \in \mathcal{F}$. 


\section{Characterizations of Transversal Matroids}

In the main part of this section, we connect Theorem 1.1 with another characterization of transversal matroids by giving a cycle of implications that proves both. While parts of the argument have entered into proofs of related results, the link between these results seems not to have been exploited before. In Section 4 we use substantial parts of the material developed here. We end this section by showing how another characterization of transversal matroids follows easily from Theorem 1.1.

To motivate the second characterization (part (3) of Theorem 3.2), we describe how to prove that a matroid $M$ that satisfies the condition in Theorem 1.1 is transversal. We want to construct a presentation of $M$. By Corollary 2.6, $M$ should have a presentation $\mathcal{A}$ in which the complement of each set $A_{i}$ is in $\mathcal{Z}(M)$. Thus, we must determine, for each $F \in \mathcal{Z}(M)$, the multiplicity of $F^{c}$ in $\mathcal{A}$. We will define a function $\beta$ on all subsets of $E(M)$ so that for each $F \in \mathcal{Z}(M)$, the multiplicity of $F^{c}$ in $\mathcal{A}$ will be $\beta(F)$. In particular, the sum of $\beta(F)$ over all $F \in \mathcal{Z}(M)$, i.e., $|\mathcal{A}|$, should be $r(M)$. By Corollary 2.3, for each $F \in \mathcal{Z}(M)$ we must have

$$
\sum_{Y \in \mathcal{Z}(M): F \cap Y^{c} \neq \emptyset} \beta(Y)=r(F),
$$

or, equivalently,

$$
\sum_{Y \in \mathcal{Z}(M): F \subseteq Y} \beta(Y)=r(M)-r(F) .
$$

With this motivation, we define $\beta$ recursively on all subsets $X$ of $E(M)$ by

$$
\beta(X)=r(M)-r(X)-\sum_{Y \in \mathcal{Z}(M): X \subset Y} \beta(Y) .
$$

By the definition of $\beta$, equation (3.2) holds whenever $F$ spans a cyclic flat of $M$. Applying that equation to the cyclic flat $\operatorname{cl}(\emptyset)$ gives

$$
\sum_{Y \in \mathcal{Z}(M)} \beta(Y)=r(M)
$$

Thus, equation (3.1) follows for $F \in \mathcal{Z}(M)$.

(The function $\beta$ is dual to the function $\alpha$ that was introduced in [14] and studied further in $[10,11]$; see the comments in the first part of Section 5. The definition of the function $\tau$ in [4] is similar to that of $\beta$, although values of $\tau$ that would otherwise be negative are set to zero; with the recursive nature of the definition, this can change the values on more sets than just those on which $\beta$ is negative. It follows from Theorem 3.2 that $\beta$ and $\tau$ agree precisely on transversal matroids.)

The next lemma plays several roles.

Lemma 3.1 If $\mathcal{F}$ is a nonempty filter in $\mathcal{Z}(M)$, then

$$
\sum_{Y \in \mathcal{F}} \beta(Y)=r(M)-\sum_{\mathcal{F}^{\prime}: \mathcal{F}^{\prime} \subseteq \mathcal{F}}(-1)^{\left|\mathcal{F}^{\prime}\right|+1} r\left(\cup \mathcal{F}^{\prime}\right) .
$$


Also, if $\mathcal{F}_{0}$ is any subset of $\mathcal{F}$ that contains every minimal set in $\mathcal{F}$, then the sum on the right can be taken just over all subsets $\mathcal{F}^{\prime}$ of $\mathcal{F}_{0}$.

Proof. For each $Y \in \mathcal{F}$, the set $\mathcal{Y}=\{F \in \mathcal{F}: F \subseteq Y\}$ is nonempty, so

$$
\sum_{\mathcal{F}^{\prime} \subseteq \mathcal{Y}: \mathcal{F}^{\prime} \neq \emptyset}(-1)^{\left|\mathcal{F}^{\prime}\right|+1}=1 .
$$

From this sum and equation (3.2), we have

$$
\begin{aligned}
\sum_{Y \in \mathcal{F}} \beta(Y) & =\sum_{Y \in \mathcal{F}} \beta(Y) \sum_{\substack{\mathcal{F}^{\prime}: \mathcal{F}^{\prime} \subseteq\{F \in \mathcal{F}: F \subseteq Y\}, \mathcal{F} \neq \emptyset \emptyset}}(-1)^{\left|\mathcal{F}^{\prime}\right|+1} \\
& =\sum_{\substack{\mathcal{F}^{\prime}: \mathcal{F}^{\prime} \subseteq \mathcal{F}, \mathcal{F}}}(-1)^{\left|\mathcal{F}^{\prime}\right|+1} \sum_{Y \in \mathcal{F}: \cup \mathcal{F}^{\prime} \subseteq Y} \beta(Y) \\
& =\sum_{\substack{\mathcal{F}^{\prime}: \mathcal{F}^{\prime} \subseteq \mathcal{F}, \mathcal{F}^{\prime} \neq \emptyset}}(-1)^{\left|\mathcal{F}^{\prime}\right|+1}\left(r(M)-r\left(\cup \mathcal{F}^{\prime}\right)\right) .
\end{aligned}
$$

Simplification yields equation (3.5). To prove the second assertion, note that for $X, Y \in \mathcal{F}$ with $X \subset Y$, the terms $(-1)^{\left|\mathcal{F}^{\prime}\right|+1} r\left(\cup \mathcal{F}^{\prime}\right)$ with $Y \in \mathcal{F}^{\prime}$ cancel via the involution that adjoins $X$ to, or omits $X$ from, $\mathcal{F}^{\prime}$.

We now turn to the first two characterizations of transversal matroids. The last part of the proof of Theorem 3.2 uses Hall's theorem: a set system $\mathcal{A}$ with $r$ sets has a transversal if and only if, for each $i \in[r]$, each union of $i$ sets in $\mathcal{A}$ has at least $i$ elements.

Theorem 3.2 For a matroid $M$, the following statements are equivalent:

(1) $M$ is transversal,

(2) for every nonempty subset (equivalently, filter; equivalently, antichain) $\mathcal{F}$ of $\mathcal{Z}(M)$,

$$
r(\cap \mathcal{F}) \leq \sum_{\mathcal{F}^{\prime} \subseteq \mathcal{F}}(-1)^{\left|\mathcal{F}^{\prime}\right|+1} r\left(\cup \mathcal{F}^{\prime}\right)
$$

(3) $\beta(X) \geq 0$ for all $X \subseteq E(M)$.

Proof. The three formulations of statement (2) are equivalent since, for $X, Y \in \mathcal{F}$ with $X \subset Y$, using $\mathcal{F}-\{Y\}$ in place of $\mathcal{F}$ preserves the right side of the inequality by the argument at the end of the proof of Lemma 3.1; also, the left side is clearly the same.

To show that statement (1) implies statement (2), extend $M$ to a fundamental transversal matroid $M_{1}$. Let $r_{1}$ and $\mathrm{cl}_{1}$ be the rank function and closure operator of $M_{1}$. For $\mathcal{F} \subseteq \mathcal{Z}(M)$, setting $\mathcal{F}_{1}=\left\{\operatorname{cl}_{1}(F): F \in \mathcal{F}\right\}$ gives $\mathcal{F}_{1} \subseteq \mathcal{Z}\left(M_{1}\right)$ as well as $r(\cup \mathcal{F})=r_{1}\left(\cup \mathcal{F}_{1}\right)$ and 
$r(\cap \mathcal{F}) \leq r_{1}\left(\cap \mathcal{F}_{1}\right)$, so statement (2) will follow by showing that for $M_{1}$, equality holds in inequality (3.6).

Let $B$ be a fundamental basis of $M_{1}$ and let $\mathcal{F} \subseteq \mathcal{Z}\left(M_{1}\right)$ be nonempty. We claim that

$$
r_{1}(\cup \mathcal{F})=|B \cap(\cup \mathcal{F})| \quad \text { and } \quad r_{1}(\cap \mathcal{F})=|B \cap(\cap \mathcal{F})| .
$$

The first equality holds since $B \cap(\cup \mathcal{F})$ is independent and each $F \in \mathcal{F}$ is spanned by $B \cap F$. For the second equality, we have $r_{1}(\cap \mathcal{F}) \geq|B \cap(\cap \mathcal{F})|$ since $B$ is independent. To show that $B \cap(\cap \mathcal{F})$ spans $\cap \mathcal{F}$, consider $x \in(\cap \mathcal{F})-B$. Since $x$ is not in the basis $B$, the set $B \cup x$ contains a unique circuit, say $C$. Clearly, $x \in C$. Similarly using the basis $B \cap F$ of $F$, for $F \in \mathcal{F}$, and the uniqueness of $C$ gives $C-x \subseteq B \cap F$; thus, $C-x \subseteq B \cap(\cap \mathcal{F})$, so, as needed, $B \cap(\cap \mathcal{F})$ spans $\cap \mathcal{F}$.

For $M_{1}$, upon using equations (3.7) to rewrite both sides of inequality (3.6), it is easy to see that equality follows from inclusion-exclusion.

We now show that statement (2) implies statement (3). For $X \subseteq E(M)$, let $\mathcal{F}(X)$ be $\{Y \in \mathcal{Z}(M): X \subset Y\}$. By equation (3.3), proving $\beta(X) \geq 0$ is the same as proving

$$
\sum_{Y \in \mathcal{F}(X)} \beta(Y) \leq r(M)-r(X) .
$$

This inequality is clear if $\mathcal{F}(X)=\emptyset$; otherwise, it follows from Lemma 3.1, statement (2), and the obvious inequality $r(X) \leq r(\cap \mathcal{F}(X))$.

Lastly, to show that statement (3) implies statement (1), we show that $M=M[\mathcal{A}]$ where $\mathcal{A}=\left(F_{1}^{c}, F_{2}^{c}, \ldots, F_{r}^{c}\right)$ is the multiset that consists of $\beta(F)$ occurrences of $F^{c}$ for each cyclic flat $F$ of $M$. By equation (3.4), we have $r=r(M)$.

To show that each dependent set $X$ of $M$ is dependent in $M[\mathcal{A}]$, it suffices to show this when $X$ is a circuit of $M$. In this case, $\operatorname{cl}_{M}(X)$ is a cyclic flat of $M$, so, by equation (3.1) and the definition of $\mathcal{A}$, it has nonempty intersection with exactly $r_{M}(X)$ sets of $\mathcal{A}$, counting multiplicity. Thus, $X$ is dependent in $M[\mathcal{A}]$ since, with $r_{M}(X)<|X|$, it cannot be a partial transversal of $\mathcal{A}$.

To show that each independent set of $M$ is independent in $M[\mathcal{A}]$, it suffices to show this for each basis $B$. For this, we use Hall's theorem to show that $\left(F_{1}^{c} \cap B, \ldots, F_{r}^{c} \cap B\right)$ has a transversal (which necessarily is $B$ ). Let $X=\bigcup_{j \in J}\left(F_{j}^{c} \cap B\right)$ with $J \subseteq[r]$. We must show $|X| \geq|J|$. Note that the cyclic flat $F_{j}$, for each $j \in J$, properly contains the independent set $B-X$, so by how $\mathcal{A}$ is defined and by statement (3) we have

$$
|J| \leq \sum_{Y \in \mathcal{Z}(M): B-X \subset Y} \beta(Y)
$$

By reformulating statement (3) as in inequality (3.8), this gives $|J| \leq r(M)-r(B-X)$, that is, $|J| \leq|B|-|B-X|$, so $|J| \leq|X|$, as needed.

It follows from equation (3.2) that the definition of $\beta$ on cyclic flats is forced by wanting a presentation in which the complement of each set is a cyclic flat. Maximal presentations have this property by Corollary 2.6, so we get the following well-known result, the first part of which we stated in Section 2. 
Corollary 3.3 The maximal presentation $\mathcal{A}$ of $M$ is unique; it consists of the sets $F^{c}$ with $F \in \mathcal{Z}(M)$, where $F^{c}$ has multiplicity $\beta(F)$ in $\mathcal{A}$.

Like Theorem 1.1, the next result is a refinement, noted by Ingleton [9], of a result of Mason [13] that used cyclic sets. Mason used this result in his proof of Theorem 1.1; we show that it follows easily from that result. Let $2^{[r]}$ be the lattice of subsets of $[r]$.

Theorem 3.4 A matroid $M$ of rank $r$ is transversal if and only if there is an injective map $\phi: \mathcal{Z}(M) \rightarrow 2^{[r]}$ with

(1) $|\phi(F)|=r(F)$ for all $F \in \mathcal{Z}(M)$,

(2) $\phi(\operatorname{cl}(F \cup G))=\phi(F) \cup \phi(G)$ for all $F, G \in \mathcal{Z}(M)$, and

(3) $r(\cap \mathcal{F}) \leq|\cap\{\phi(F): F \in \mathcal{F}\}|$ for every subset (equivalently, filter; equivalently, antichain) $\mathcal{F}$ of $\mathcal{Z}(M)$.

Proof. Assume $M=M[\mathcal{A}]$ with $\mathcal{A}=\left(A_{1}, A_{2}, \ldots, A_{r}\right)$. For $F \in \mathcal{Z}(M)$, let

$$
\phi(F)=\left\{k: F \cap A_{k} \neq \emptyset\right\} .
$$

It is easy to see that $\phi$ is an injection and that properties (1)-(3) hold; in particular, the first is Corollary 2.3. For the converse, assume $\phi: \mathcal{Z}(M) \rightarrow 2^{[r]}$ is an injection that satisfies properties (1)-(3). For any nonempty subset $\mathcal{F}$ of $\mathcal{Z}(M)$, properties (1) and (2) allow us to recast the right side of inequality (3.6) as the summation part of an inclusion-exclusion equation for the sets $\phi(F)$ with $F \in \mathcal{F}$; inequality (3.6) follows from inclusion-exclusion and property (3), so $M$ is transversal by Theorem 3.2.

\section{Characterizations of Fundamental Transversal Matroids}

In this section, we treat counterparts, for fundamental transversal matroids, of the results in the last section. In contrast to Theorem 1.1, in the main result, Theorem 4.1, we must work with cyclic flats since equality (4.1) may fail for sets $\mathcal{F}$ of cyclic sets.

Theorem 4.1 A matroid $M$ is a fundamental transversal matroid if and only if

$$
r(\cap \mathcal{F})=\sum_{\mathcal{F}^{\prime} \subseteq \mathcal{F}}(-1)^{\left|\mathcal{F}^{\prime}\right|+1} r\left(\cup \mathcal{F}^{\prime}\right)
$$

for all nonempty subsets (equivalently, antichains; equivalently, filters) $\mathcal{F} \subseteq \mathcal{Z}(M)$.

In the proof of Theorem 3.2, we showed that equation (4.1) holds for all fundamental transversal matroids; below we prove the converse. In the proof, we use the notation $\Delta(x)$ and $\Delta(X)$ that we defined in Section 2. The following well-known lemma is easy to prove. 
Lemma 4.2 For any presentation of a transversal matroid $M$, if $C$ is a circuit of $M$, then $\Delta(C)=\Delta(C-x)$ for all $x \in C$.

By Theorem 3.2, if equation (4.1) always holds, then $M$ is transversal. In this setting, the next lemma identifies $|\Delta(x)|$, for the maximal presentation, as the rank of a set.

Lemma 4.3 Let $\left(A_{1}, A_{2}, \ldots, A_{r}\right)$ be the maximal presentation of a transversal matroid $M$ for which equality (4.1) holds for all nonempty subsets of $\mathcal{Z}(M)$. For each $x \in E(M)$, we have $|\Delta(x)|=r(\cap \mathcal{F})$ where $\mathcal{F}=\{F \in \mathcal{Z}(M): x \in F\}$.

Proof. The set $\Delta(x)$ contains the vertices $v_{k}$ where $A_{k}=F^{c}$ and $F \in \mathcal{Z}(M)-\mathcal{F}$. By Lemma 3.1, Corollary 3.3, and equations (3.4) and (4.1), $|\Delta(x)|$ is, as stated,

$$
\sum_{F \in \mathcal{Z}(M)-\mathcal{F}} \beta(F)=r(\cap \mathcal{F}) .
$$

The equality $|\Delta(x)|=r(\cap \mathcal{F})$ may fail if equality (4.1) fails. For example, consider the rank-4 matroid on $\{a, b, c, d, e, f, g\}$ in which $\{a, b, c, d\}$ and $\{d, e, f, g\}$ are the only nonspanning circuits. In the affine representation arising from the maximal presentation, $d$ is placed freely on an edge of the simplex even though the cyclic flats that contain it intersect in rank one.

We now prove the main result.

Proof of Theorem 4.1. Assume equation (4.1) holds for all nonempty sets of cyclic flats. As noted above, $M$ is transversal. Coloops can be placed at vertices of $\Delta$ and doing so reduces the problem to a smaller one, so we may assume that $M$ has no coloops. Thus, $E(M) \in \mathcal{Z}(M)$. The set $V$ of vertices of $\Delta$ has size $r(M)$, so $\Delta(E(M))=V$.

Let $\mathcal{A}$ be the maximal presentation of $M$. As parts (a) and (c) of Figure 1 show, from the corresponding affine representation, it may be possible to get other affine representations of $M$ by moving some elements of $M$ to vertices of $\Delta$, where $x \in E(M)$ may be moved only to a vertex in $\Delta_{\mathcal{A}}(x)$. Such affine representations correspond to presentations $\mathcal{A}^{\prime}$ of $M$ in which, for each $x \in E(M)$, either $\Delta_{\mathcal{A}^{\prime}}(x)=\Delta_{\mathcal{A}}(x)$ or $\Delta_{\mathcal{A}^{\prime}}(x)=\left\{v_{i}\right\}$ for some $v_{i} \in \Delta_{\mathcal{A}}(x)$. Among all such affine representations, fix one with the minimum number of vertices of $\Delta$ at which no element of $E(M)$ is placed; let $\mathcal{A}^{\prime}$ be the corresponding presentation. To show that $M$ is fundamental, we show that if, in this affine representation, no element is placed at vertex $v_{i}$ of $\Delta$, then we get another affine representation of $M$ by moving some element there, which contradicts the minimality assumption.

To show this, we will use the fundamental transversal matroid $M_{1}$ that we obtain from the fixed affine representation of $M$ (corresponding to $\mathcal{A}^{\prime}$ ) by adding an element (which we call $v_{j}$ ) at each vertex $v_{j}$ of $\Delta$ at which there is no element of $M$. Let $\mathcal{P}$ be the corresponding presentation of $M_{1}$. Let $r_{1}$ and $\mathrm{cl}_{1}$ be its rank function and closure operator. For $\mathcal{F} \subseteq \mathcal{Z}(M)$, let $\mathcal{F}_{1}=\left\{\operatorname{cl}_{1}(F): F \in \mathcal{F}\right\}$. Clearly $r(\cup \mathcal{F})=r_{1}\left(\cup \mathcal{F}_{1}\right)$. We claim that

(i) $r(\cap \mathcal{F})=r_{1}\left(\cap \mathcal{F}_{1}\right)$,

(ii) $\Delta_{\mathcal{A}^{\prime}}(\cap \mathcal{F})=\Delta_{\mathcal{P}}\left(\cap \mathcal{F}_{1}\right)$, and 
(iii) $r(\cap \mathcal{F})=\left|\Delta_{\mathcal{A}^{\prime}}(\cap \mathcal{F})\right|$.

To prove these properties, note that since $M_{1}$ is fundamental, we have

$$
\sum_{\mathcal{F}^{\prime} \subseteq \mathcal{F}_{1}}(-1)^{\left|\mathcal{F}^{\prime}\right|+1} r_{1}\left(\cup \mathcal{F}^{\prime}\right)=r_{1}\left(\cap \mathcal{F}_{1}\right)
$$

Term by term, the sum agrees with its counterpart for $\mathcal{F}$ in $M$, so property (i) follows from equation (4.1). Clearly, $r(\cap \mathcal{F}) \leq\left|\Delta_{\mathcal{A}^{\prime}}(\cap \mathcal{F})\right|$. Also, $\Delta_{\mathcal{A}^{\prime}}(\cap \mathcal{F}) \subseteq \Delta_{\mathcal{P}}\left(\cap \mathcal{F}_{1}\right)$ since $\cap \mathcal{F}_{1}$ contains $\cap \mathcal{F}$. Since $M_{1}$ is fundamental, equation (3.7) holds, from which we get $\left|\Delta_{\mathcal{P}}\left(\cap \mathcal{F}_{1}\right)\right|=r_{1}\left(\cap \mathcal{F}_{1}\right)$. With these deductions, property (i) gives properties (ii) and (iii).

Now assume that no element of $M$ has been placed at vertex $v_{i}$ of $\Delta$. Let

$$
\mathcal{F}=\left\{F \in \mathcal{Z}(M): v_{i} \in \Delta_{\mathcal{A}^{\prime}}(F)\right\}
$$

(By Corollary 2.3, $\Delta_{\mathcal{A}}(F)=\Delta_{\mathcal{A}^{\prime}}(F)$ for all $F \in \mathcal{Z}(M)$.) Now $E(M) \in \mathcal{F}$, so $\mathcal{F} \neq \emptyset$. Since $v_{i}$ is in all sets in $\mathcal{F}_{1}$, we get $v_{i} \in \Delta_{\mathcal{P}}\left(\cap \mathcal{F}_{1}\right)$, so property (ii) gives $v_{i} \in \Delta_{\mathcal{A}^{\prime}}(\cap \mathcal{F})$. Fix $x \in \cap \mathcal{F}$ with $v_{i} \in \Delta_{\mathcal{A}^{\prime}}(x)$.

We claim that $\mathcal{F}=\{F \in \mathcal{Z}(M): x \in F\}$. If $F \in \mathcal{F}$, then $\cap \mathcal{F} \subseteq F$, so $x \in F$. Conversely, if $F \in \mathcal{Z}(M)$ and $x \in F$, then $v_{i} \in \Delta_{\mathcal{A}^{\prime}}(F)$ since $\Delta_{\mathcal{A}^{\prime}}(x) \subseteq \Delta_{\mathcal{A}^{\prime}}(F)$.

Now $v_{i} \in \Delta_{\mathcal{A}^{\prime}}(x)$ but $x$ was not placed at $v_{i}$, so $\Delta_{\mathcal{A}^{\prime}}(x)=\Delta_{\mathcal{A}}(x)$. Since $x \in \cap \mathcal{F}$, we have $\Delta_{\mathcal{A}^{\prime}}(x) \subseteq \Delta_{\mathcal{A}^{\prime}}(\cap \mathcal{F})$; property (iii), the previous paragraph, and Lemma 4.3 give equality, that is, $x$ is placed freely in the face $\Delta_{\mathcal{A}^{\prime}}(\cap \mathcal{F})$. Let $M_{2}$ be the matroid that is obtained by moving $x$ to $v_{i}$, that is, $M_{2}=M\left[\mathcal{A}^{\prime \prime}\right]$ where $\mathcal{A}^{\prime \prime}$ is formed from $\mathcal{A}^{\prime}$ by removing $x$ from all sets except the one indexed by $i$. We claim that $M$ and $M_{2}$ have the same circuits and so are the same matroid, thus proving our claim that some element can be moved to $v_{i}$. Among all sets $C$ that are circuits of just one of $M$ and $M_{2}$ (if there are any), let $C$ have minimum size. Clearly, $x \in C$.

We claim that $\Delta_{\mathcal{A}^{\prime}}(C)=\Delta_{\mathcal{A}^{\prime \prime}}(C)$. If $C$ is a circuit of $M$, then the claim follows from Lemma 4.2, the inclusion $\Delta_{\mathcal{A}^{\prime \prime}}(x) \subset \Delta_{\mathcal{A}^{\prime}}(x)$, and the observation that $\Delta_{\mathcal{A}^{\prime}}(y)=\Delta_{\mathcal{A}^{\prime \prime}}(y)$ for $y \in C-x$. Assume $C$ is a circuit of $M_{2}$. By Lemma 4.2, $v_{i} \in \Delta_{\mathcal{A}^{\prime \prime}}(y)$ for some $y$ in $C-x$. Thus, $v_{i} \in \Delta_{\mathcal{A}^{\prime}}(y)$, so all cyclic flats that contain $y$ are in $\mathcal{F}$ and so contain $x$; thus, all sets in the maximal presentation that contain $x$ also contain $y$, that is, $\Delta_{\mathcal{A}}(x) \subseteq \Delta_{\mathcal{A}}(y)$. Since no element prior to $x$ was placed at $v_{i}$, we have $\Delta_{\mathcal{A}^{\prime}}(y)=\Delta_{\mathcal{A}}(y)$; also, as noted above, $\Delta_{\mathcal{A}^{\prime}}(x)=\Delta_{\mathcal{A}}(x)$, so $\Delta_{\mathcal{A}^{\prime}}(x) \subseteq \Delta_{\mathcal{A}^{\prime}}(y)$, from which the claim follows.

Now $C$ is a circuit in one of $M$ and $M_{2}$, so, since $\Delta_{\mathcal{A}^{\prime}}(C)=\Delta_{\mathcal{A}^{\prime \prime}}(C)$, we have

$$
\left|\Delta_{\mathcal{A}^{\prime}}(C)\right|=\left|\Delta_{\mathcal{A}^{\prime \prime}}(C)\right|<|C|
$$

It follows that $C$ is dependent in both $M$ and $M_{2}$. From this conclusion and the minimality assumed for $|C|$, it follows that $C$ cannot be a circuit of just one of $M$ and $M_{2}$. Thus, $M$ and $M_{2}$ have the same circuits and so are the same matroid, as we needed to show.

The following result is immediate from Theorem 4.1 and Lemma 3.1. 
Theorem 4.4 A matroid $M$ is a fundamental transversal matroid if and only if

$$
\sum_{Y \in \mathcal{F}} \beta(Y)=r(M)-r(\cap \mathcal{F})
$$

for all filters $\mathcal{F} \subseteq \mathcal{Z}(M)$.

The proof of the next result is similar to that of Theorem 3.4 and uses Theorem 4.1.

Theorem 4.5 A matroid $M$ of rank $r$ is a fundamental transversal matroid if and only if there is an injection $\phi: \mathcal{Z}(M) \rightarrow 2^{[r]}$ with

(1) $|\phi(F)|=r(F)$ for all $F \in \mathcal{Z}(M)$,

(2) $\phi(\operatorname{cl}(F \cup G))=\phi(F) \cup \phi(G)$ for all $F, G \in \mathcal{Z}(M)$, and

(3) $r(\cap \mathcal{F})=|\cap\{\phi(F): F \in \mathcal{F}\}|$ for every subset (equivalently, filter; equivalently, antichain) $\mathcal{F}$ of $\mathcal{Z}(M)$.

If the matroid $M$ is already known to be transversal and if a presentation of $M$ is known, then we should define the function $\phi$ in the last result as in equation (3.9) or, equivalently, $\phi(F)=\left\{k: v_{k} \in \Delta(F)\right\}$. Properties (1) and (2) then hold, so we have the next corollary.

Corollary 4.6 Let $\mathcal{A}$ be any presentation of a transversal matroid $M$. The matroid $M$ is fundamental if and only if $r(\cap \mathcal{F})=|\cap\{\Delta(F): F \in \mathcal{F}\}|$ for every subset (equivalently, filter; equivalently, antichain) $\mathcal{F}$ of $\mathcal{Z}(M)$.

\section{Observations and Applications}

We first consider the duals of the results above. In particular, Theorem 5.1 makes precise the remark before Lemma 3.1, that $\beta$ is dual to Mason's function $\alpha$; this shows that the equivalence of statements (1) and (3) in Theorem 3.2 is the dual of Mason's result that $M$ is a cotransversal matroid (a strict gammoid) if and only if $\alpha(X) \geq 0$ for all $X \subseteq E(M)$.

It is well known and easy to prove that

$$
\mathcal{Z}\left(M^{*}\right)=\{E(M)-F: F \in \mathcal{Z}(M)\},
$$

where $M^{*}$ is the dual of $M$. With this result and the formula

$$
r^{*}(X)=|X|-r(M)+r(E(M)-X)
$$

for the rank function $r^{*}$ of $M^{*}$, it is routine to show that a matroid $M$ satisfies statement (2) in Theorem 3.2 if and only if for all sets (equivalently, all ideals; equivalently, all antichains) $\mathcal{F} \subseteq \mathcal{Z}\left(M^{*}\right)$,

$$
r^{*}(\cup \mathcal{F}) \leq \sum_{\mathcal{F}^{\prime} \subseteq \mathcal{F}: \mathcal{F}^{\prime} \neq \emptyset}(-1)^{\left|\mathcal{F}^{\prime}\right|+1} r^{*}\left(\cap \mathcal{F}^{\prime}\right) .
$$


Thus, this condition characterizes cotransversal matroids $M^{*}$.

We now recall the function $\alpha$ that Mason introduced in [14], which is defined recursively as follows. For $X \subseteq E(M)$, set

$$
\alpha(X)=\eta(X)-\sum_{\text {flats } F: F \subset X} \alpha(F)
$$

where $\eta(X)$ is the nullity, $|X|-r(X)$, of $X$. Thus for any flat $X$ of $M$,

$$
\sum_{\text {flats } F: F \subseteq X} \alpha(F)=\eta(X)
$$

To prepare to link the functions $\alpha$ and $\beta$, we first show that $\alpha(F)=0$ if $F$ is a noncyclic flat. Induct on $|F|$. The base case holds vacuously. Let $I$ be the set of coloops of $M \mid F$ and set $F^{\prime}=F-I$, so $F^{\prime} \in \mathcal{Z}(M)$. Since $\eta(F)=\eta\left(F^{\prime}\right)$, equation (5.4) gives

$$
\sum_{\text {flats } Y: Y \subseteq F} \alpha(Y)=\sum_{\text {flats } Y^{\prime}: Y^{\prime} \subseteq F^{\prime}} \alpha\left(Y^{\prime}\right) .
$$

Now $F$ and $F^{\prime}$ contain precisely the same cyclic flats, so $\alpha(F)$ is the only term in which the two sides of this equality differ that is not yet known to be zero, so $\alpha(F)=0$.

It now follows that the sum in equation (5.3) can be over just $F \in \mathcal{Z}(M)$ with $F \subset X$. With induction, the next theorem follows from this result and equations (5.1) and (5.2).

Theorem 5.1 For any matroid $M$, if $X \subseteq E(M)$, then $\alpha_{M}(X)=\beta_{M^{*}}(E(M)-X)$.

As shown in [12], the class of fundamental transversal matroids is closed under duality. (To see this, note that a basis $B$ of $M$ is fundamental if and only if $r(M)=r(F)+|B-F|$ for every $F \in \mathcal{Z}(M)$; a routine rank calculation then shows that $B$ is a fundamental basis of $M$ if and only if $E(M)-B$ is a fundamental basis of $M^{*}$.) Using this result and those above, it is easy to deduce the following dual versions of Theorems 4.1 and 4.4. (Likewise, one can dualize Theorem 4.5 and Corollary 4.6.)

Theorem 5.2 For a matroid $M$, the following statements are equivalent:

(1) $M$ is a fundamental transversal matroid,

(2) for all subsets (equivalently, ideals; equivalently, antichains) $\mathcal{F}$ of $\mathcal{Z}(M)$,

$$
r(\cup \mathcal{F})=\sum_{\mathcal{F}^{\prime} \subseteq \mathcal{F}: \mathcal{F}^{\prime} \neq \emptyset}(-1)^{\left|\mathcal{F}^{\prime}\right|+1} r\left(\cap \mathcal{F}^{\prime}\right),
$$

(3) for all ideals $\mathcal{F} \subseteq \mathcal{Z}(M)$,

$$
\sum_{Y \in \mathcal{F}} \alpha(Y)=\eta(\cup \mathcal{F})
$$


We now consider how the results above extend to transversal matroids of finite rank on infinite sets. Although the ground set is infinite, every multiset we consider is finite. Thus, let $M$ be $M[\mathcal{A}]$ where $\mathcal{A}=\left(A_{1}, A_{2}, \ldots, A_{r}\right)$ is a set system on the infinite set $E(M)$. For each subset $X$ of $E(M)$, let $\phi(X)=\left\{k: X \cap A_{k} \neq \emptyset\right\}$. It is easy to see that if $F$ is a cyclic flat of $M$, then $F=\{x: \phi(x) \subseteq \phi(F)\}$. It follows that $M$ has at most $\left(\begin{array}{l}r \\ k\end{array}\right)$ cyclic flats of rank $k$, so $\mathcal{Z}(M)$ is a finite lattice. Whenever $M$ has finite rank and $\mathcal{Z}(M)$ is finite, the definition of $\beta$ makes sense, as do the sums that appear in the results above. Reviewing the proofs shows that Theorems 3.2, 3.4, 4.1, 4.4, 4.5, and Corollary 4.6 hold in this setting, where we add to the hypotheses of all but the last result the requirements that $M$ has finite rank and $\mathcal{Z}(M)$ is finite. Note that in this setting, the assertion that matroids with fundamental bases are transversal holds since the argument proving statement (2) in Theorem 3.2 shows that such matroids satisfy that statement (with equality). In contrast, Theorem 5.2 was obtained by duality, which does not apply within the class of matroids of finite rank on infinite sets. However, we have the following result.

Theorem 5.3 A matroid $M$ of finite rank on an infinite set is a fundamental transversal matroid if and only if the lattice $\mathcal{Z}(M)$ is finite and equation (5.5) holds for all of its subsets (equivalently, ideals; equivalently, antichains).

Proof. First assume $M$ is a fundamental transversal matroid. Let $X$ be a finite subset of $E(M)$ whose subsets include a fundamental basis, a cyclic spanning set for each cyclic flat, and a spanning set for each intersection of cyclic flats. It follows that $M \mid X$ is a fundamental transversal matroid and the map $\psi: \mathcal{Z}(M \mid X) \rightarrow \mathcal{Z}(M)$ given by $\psi(Y)=\mathrm{cl}_{M}(Y)$ is a rank-preserving isomorphism. Since $M \mid X$ is fundamental, the counterpart of equation (5.5) holds for $M \mid X$. By using $\psi$, we can deduce equation (5.5) for $M$.

To prove the converse, let $r=r(M)$ and let $X$ be a finite subset of $E(M)$ that contains a cyclic spanning set for each cyclic flat and a spanning set for each intersection of cyclic flats. As above, the map $\psi$ given by $\psi(Y)=\mathrm{cl}_{M}(Y)$ is a rank-preserving isomorphism of $\mathcal{Z}(M \mid X)$ onto $\mathcal{Z}(M)$. Using $\psi$, from the validity of equation (5.5) for $M$ we can deduce its counterpart for $M \mid X$, so $M \mid X$ is fundamental by Theorem 5.2. Thus, some injection $\phi: \mathcal{Z}(M \mid X) \rightarrow 2^{[r]}$ satisfies properties (1)-(3) of Theorem 4.5. Define $\phi^{\prime}: \mathcal{Z}(M) \rightarrow 2^{[r]}$ by $\phi^{\prime}(F)=\phi(F \mid X)$. It is immediate that $\phi^{\prime}$ is an injection that satisfies properties (1)-(3) of Theorem 4.5 , so $M$ is fundamental.

Brylawski's characterization of fundamental transversal matroids [5, Proposition 4.2], which we state next, follows easily from Theorem 5.2.

Theorem 5.4 A matroid $M$ is a fundamental transversal matroid if and only if for all families $\mathcal{F}$ of intersections of cyclic flats,

$$
r(\cup \mathcal{F}) \geq \sum_{\mathcal{F}^{\prime} \subseteq \mathcal{F}: \mathcal{F}^{\prime} \neq \emptyset}(-1)^{\left|\mathcal{F}^{\prime}\right|+1} r\left(\cap \mathcal{F}^{\prime}\right),
$$

or, equivalently, equality holds in inequality (5.6). The same statement holds for matroids of finite rank on infinite sets where, in the second part, we add that $\mathcal{Z}(M)$ is finite. 
Proof. An inclusion-exclusion argument like that in the proof of Theorem 3.2 shows that equality holds in inequality (5.6) for fundamental transversal matroids. For the converse, note that if that equality always holds, then $M$ is fundamental by Theorem 5.2. Thus, it suffices to show that having inequality (5.6) hold for all families $\mathcal{F}$ of intersections of cyclic flats of $M$ yields equality. To prove this, we induct on $|\mathcal{F}|$. The base case, $|\mathcal{F}|=1$, is obvious. Assume that $|\mathcal{F}|>1$ and that equality holds for all families of intersections of cyclic flats that have fewer sets than $\mathcal{F}$. Fix $X \in \mathcal{F}$ and let $\mathcal{F}_{\widehat{X}}=\mathcal{F}-\{X\}$. The set $\mathcal{F}^{\prime}=\{X\}$ contributes $r(X)$ to the sum. The sets $\mathcal{F}^{\prime}$ with $\mathcal{F}^{\prime} \subseteq \mathcal{F}_{\widehat{X}}$ give terms that, by the induction hypothesis, together contribute $r\left(\cup \mathcal{F}_{\widehat{X}}\right)$ to the sum. The sets $\mathcal{F}^{\prime}$ with $\{X\} \subset \mathcal{F}^{\prime}$ contribute terms that are the negatives of the terms in the corresponding sum based on the family $\left\{F \cap X: F \in \mathcal{F}_{\widehat{X}}\right\}$; by the induction hypothesis, the sum of these terms is $-r\left(X \cap\left(\cup \mathcal{F}_{\widehat{X}}\right)\right)$. Thus, inequality (5.6) is equivalent to

$$
r(\cup \mathcal{F}) \geq r(X)+r\left(\cup \mathcal{F}_{\widehat{X}}\right)-r\left(X \cap\left(\cup \mathcal{F}_{\widehat{X}}\right)\right)
$$

Semimodularity (the opposite inequality) gives equality. This completes the induction.

Finally, we apply our results to the free product, which was introduced and studied by Crapo and Schmitt $[6,7]$. Given matroids $M$ and $N$ on disjoint sets, their free product $M \square N$ is the matroid on the set $E(M) \cup E(N)$ whose bases are the subsets $B$ with (i) $|B|=r(M)+r(N)$, (ii) $B \cap E(M)$ independent in $M$, and (iii) $B \cap E(N)$ spanning $N$ (see [7, Proposition 3.3]). In general, $M \square N \neq N \square M$. Relative to the weak order, the free product is the greatest matroid $M^{\prime}$ on $E(M) \cup E(N)$ with $M^{\prime} \backslash E(N)=M$ and $M^{\prime} / E(M)=N$. Special cases of the free product include the free extension of $M$ (set $N=U_{0,1}$ ) and the free coextension of $N$ (set $\left.M=U_{1,1}\right)$. The dual of the free product is given by $(M \square N)^{*}=N^{*} \square M^{*}$. The following result is [7, Proposition 6.1].

Proposition 5.5 A subset $F$ of $E(M) \cup E(N)$ other than $E(M)$ is in $\mathcal{Z}(M \square N)$ if and only if either (i) $F \subset E(M)$ and $F \in \mathcal{Z}(M)$ or (ii) $E(M) \subset F$ and $F-E(M) \in \mathcal{Z}(N)$. The set $E(M)$ is in $\mathcal{Z}(M \square N)$ if and only if $E(M) \in \mathcal{Z}(M)$ and $\emptyset \in \mathcal{Z}(N)$.

By giving a presentation of $M \square N$ from presentations of $M$ and $N$, Crapo and Schmitt [7, Proposition 4.14] showed that free products of transversal matroids are transversal. The following extension of their result can be proven using either ideas in [7] or, as we show below, Theorems 3.2 and 4.1 .

Theorem 5.6 For matroids $M$ and $N$ on disjoint ground sets, their free product $M \square N$ is transversal if and only if both $M$ and $N$ are. The corresponding statements hold for fundamental transversal matroids, for cotransversal matroids, and for matroids that are both transversal and cotransversal.

Proof. The proof of each part uses one of Theorems 3.2 and 4.1 along with three observations:

(1) by Proposition 5.5, any antichain in $\mathcal{Z}(M \square N)$ is either (i) an antichain in $\mathcal{Z}(M)$ or (ii) obtained from an antichain in $\mathcal{Z}(N)$ by augmenting each set by $E(M)$; 
(2) if $X \subseteq E(M)$, then $r_{M \square N}(X)=r_{M}(X)$ since $(M \square N) \backslash E(N)=M$;

(3) if $X \subseteq E(N)$, then $r_{M \square N}(X \cup E(M))=r_{N}(X)+r(M)$ since $(M \square N) / E(M)$ is $N$.

To illustrate the argument, assume that $M$ and $N$ are fundamental transversal matroids and let $\mathcal{F}$ be an antichain in $\mathcal{Z}(M \square N)$. If $\mathcal{F}$ is an antichain in $\mathcal{Z}(M)$, then equation (4.1) holds using the rank function of $M$, so, by observation (2), this equation also holds for $\mathcal{F}$ using the rank function of $M \square N$. Assume instead that $\mathcal{F}$ is obtained by augmenting, by $E(M)$, each set in an antichain $\mathcal{F}_{N}$ in $\mathcal{Z}(N)$. Note that the equality we know, namely,

$$
r_{N}\left(\cap \mathcal{F}_{N}\right)=\sum_{\mathcal{F}^{\prime} \subseteq \mathcal{F}_{N}}(-1)^{\left|\mathcal{F}^{\prime}\right|+1} r_{N}\left(\cup \mathcal{F}^{\prime}\right),
$$

is preserved if we replace $r_{N}\left(\cap \mathcal{F}_{N}\right)$ by $r_{N}\left(\cap \mathcal{F}_{N}\right)+r(M)$ and, when $\mathcal{F}^{\prime} \neq \emptyset$, replace $r_{N}\left(\cup \mathcal{F}^{\prime}\right)$ by $r_{N}\left(\cup \mathcal{F}^{\prime}\right)+r(M)$. Thus, by observation (3), equation (4.1) holds for the antichain $\mathcal{F}$ in $\mathcal{Z}(M \square N)$. Therefore $M \square N$ is a fundamental transversal matroid. The proofs of the converse and the remaining assertions are similar.

\section{Acknowledgement}

The authors thank the referee for useful feedback on the exposition.

\section{References}

[1] J. Bonin, A construction of infinite sets of intertwines for pairs of matroids, SIAM J. Discrete Math. 24 (2010) 1742-1752.

[2] J. Bonin, Transversal lattices, Electron. J. Combin. 15 (2008) Research Paper 15, 11 pp.

[3] J. Bonin and A. de Mier, The lattice of cyclic flats of a matroid, Ann. Comb. 12 (2008) $155-170$.

[4] R.A. Brualdi, Transversal matroids, in: Combinatorial Geometries, N. White, ed. (Cambridge Univ. Press, Cambridge, 1987) 72-97.

[5] T.H. Brylawski, An affine representation for transversal geometries, Studies in Appl. Math. 54 (1975) 143-160.

[6] H.H. Crapo and W.R. Schmitt, The free product of matroids, European J. Combin. 26 (2005) 1060-1065.

[7] H.H. Crapo and W.R. Schmitt, A unique factorization theorem for matroids, J. Combin. Theory Ser. A 112 (2005) 222-249.

[8] J. Edmonds and D.R. Fulkerson, Transversals and matroid partition, J. Res. Nat. Bur. Standards Sect. B 69B (1965) 147-153.

[9] A.W. Ingleton, Transversal matroids and related structures, in: Higher Combinatorics, M. Aigner, ed. (Proc. NATO Advanced Study Inst., Berlin, 1976; Reidel, DordrechtBoston, MA, 1977) 117-131. 
[10] A.W. Ingleton and M.J. Piff, Gammoids and transversal matroids, J. Combin. Theory Ser. B 15 (1973) 51-68.

[11] J.P.S. Kung, The alpha function of a matroid, I: Transversal matroids, Studies in Appl. Math. 58 (1978) 263-275.

[12] M. Las Vergnas, Sur les systèmes de représentants distincts d'une famille d'ensembles, $C$. R. Acad. Sci. Paris Sér. A-B 270 (1970) A501-A503.

[13] J.H. Mason, A characterization of transversal independence spaces, in: Théorie des Matroïdes (Lecture Notes in Math., Vol. 211, Springer, Berlin, 1971) 86-94.

[14] J.H. Mason, On a class of matroids arising from paths in graphs, Proc. London Math. Soc. 25 (1972) 55-74.

[15] J.G. Oxley, Matroid Theory, (Oxford University Press, Oxford, 1992).

[16] D.J.A. Welsh, Matroid Theory, (Academic Press, London, 1976). 